\title{
Fractional and $j$-Fold Coloring of the Plane
}

\author{
Jarosław Grytczuk ${ }^{1,2}$. \\ Konstanty Junosza-Szaniawski² ${ }^{2}$ Joanna Sokót ${ }^{2}$. \\ Krzysztof Wesek ${ }^{2}$
}

Received: 23 December 2014 / Revised: 9 February 2016 / Accepted: 16 February 2016 /

Published online: 8 March 2016

(C) The Author(s) 2016. This article is published with open access at Springerlink.com

\begin{abstract}
We present results referring to the Hadwiger-Nelson problem which asks for the minimum number of colors needed to color the plane with no two points at distance 1 having the same color. Exoo considered a more general problem concerning graphs $G_{[a, b]}$ with $\mathbb{R}^{2}$ as the vertex set and two vertices adjacent if their distance is in the interval $[a, b]$. Exoo conjectured $\chi\left(G_{[a, b]}\right)=7$ for sufficiently small but positive difference between $a$ and $b$. We partially answer this conjecture by proving that $\chi\left(G_{[a, b]}\right) \geqslant 5$ for $b>a$. A $j$-fold coloring of a graph $G=(V, E)$ is an assignment of $j$-elemental sets of colors to the vertices of $G$, in such a way that the sets assigned to any two adjacent vertices are disjoint. The fractional chromatic number $\chi_{f}(G)$ is the infimum of fractions $k / j$ for $j$-fold coloring of $G$ using $k$ colors. We generalize a method by Hochberg and O'Donnel (who proved that $G_{[1,1]} \leqslant 4.36$ ) for the fractional coloring of graphs $G_{[a, b]}$, obtaining a bound dependent on $\frac{a}{b}$. We also present few specific and two general methods for $j$-fold coloring of $G_{[a, b]}$ for small $j$, in particular for $G_{[1,1]}$ and $G_{[1,2]}$. The $j$-fold coloring for small $j$ has strong practical
\end{abstract}

Editor in Charge: János Pach

Jarosław Grytczuk

j.grytczuk@mini.pw.edu.pl

Konstanty Junosza-Szaniawski

k.szaniawski@mini.pw.edu.pl

Joanna Sokół

j.sokol@mini.pw.edu.pl

Krzysztof Węsek

k.wesek@mini.pw.edu.pl

1 Faculty of Mathematics and Computer Science, Jagiellonian University, Kraków, Poland

2 Faculty of Mathematics and Information Science, Warsaw University of Technology, Warsaw, Poland 
motivation especially in scheduling theory, while graph $G_{[1,2]}$ is often used to model hidden conflicts in radio networks.

Keywords Fractional coloring $\cdot$ Hadwiger-Nelson problem $\cdot$ Coloring of the plane

Mathematics Subject Classification $\quad 05 \mathrm{C} 15 \cdot 05 \mathrm{C} 10 \cdot 05 \mathrm{C} 62$

\section{Introduction}

\subsection{Mathematical Context}

The famous Hadwiger-Nelson problem asks for the minimum number of colors required to color the Euclidean plane $\mathbb{R}^{2}$ in such a way that no two points at distance 1 from each other have the same color. The question can be equivalently stated in the graph theory language: Recall that a coloring of a graph $G=(V, E)$ is a function $c: V \rightarrow K$ (where $K$ is a set of colors) such that every two adjacent vertices $x, y$ satisfy $c(x) \neq c(y)$. The chromatic number $\chi(G)$ of $G$ is the minimal cardinality of the set of colors to color $G$. Therefore, the Hadwiger-Nelson problem is about determining the chromatic number of the graph on the set of vertices $\mathbb{R}^{2}$ with vertices in distance one adjacent-it is called unit distance graph and in this article will be denoted by $G_{[1,1]}$ (the notation will be justified shortly).

The problem was originally proposed by Edward Nelson in 1950 and was made publicly known by Hugo Hadwiger [4]. Pioneers on the topic observed the following bounds: Nelson first showed that at least 4 colors are needed (see the proof by Moser and Moser [7] which uses the so-called Moser spindle) and John Isbell was first to prove that 7 colors are enough (this result was published by Hadwiger [4]). Somehow surprisingly, the aforementioned bounds remain unchanged since their origin in 1950s, i.e. for more than 60 years nobody has found anything sharper than $4 \leqslant \chi(G) \leqslant 7$-as long as we consider the full generality. Nevertheless, advanced studies on the question, its subproblems and other related topics provide some understanding. For example, if we consider only measurable colorings (i.e. with measurable colors) then at least 5 colors are necessary [2] and if coloring of the plane consists of regions bounded by Jordan curves then at least 6 colors are required [12]. Generally, across the decades the Hadwiger-Nelson problem inspired many interesting results in the touchpoint of combinatorics and geometry, a vast number of challenging problems and various applications [10].

One of the possible ways to generalize the first question was presented by Exoo [1] (see also [6]). He considered graphs on the set of vertices $\mathbb{R}^{2}$ with vertices in distance in the interval $[a, b]$, we denote such graphs by $G_{[a, b]}$. How many colors would be enough to color such a graph, depending on $a$ and $b$ ? Are there some important ranges of those parameters?

The other path for research leading from the Hadwiger-Nelson problem concerns different models of graphs coloring. In the majority of this article, we investigate fractional colorings, in some sense a generalization of the classic coloring: every vertex gets a $j$-elemental set of colors from the set of colors (of size $k$ ) and the sets for 
adjacent vertices have to be disjoint. The 'quality' of such a coloring is measured by the fraction $k / j$ and the fractional chromatic number is the infimum of such fractions (one can also ask for the best fraction with a fixed $j$ ). It can be seen as in this case every single color is just a $1 / j$-size part of a 'complete color', so we divide 'complete colors' from the classic coloring to somehow save a bit by combining partitioned colors in a clever way. It turns out that in fact we can save much using fractional coloring: fractional chromatic number is always lower or equal to chromatic number of a given graph, but the difference can be arbitrarily large.

The fractional chromatic number of the graph $G_{[1,1]}$ has been studied in the literature. The best upper bound is due to Hochbeg and O'Donnell [5] (based on an idea by Fisher and Ullman [3] of looking for a dense subset of the plane which avoids unit distance) and the best lower bound (using a finite subgraph of the plane) can be found in the book of Scheinerman and Ullman [9] (along with a good explanation of the upper bound): $3.555 \leqslant \chi_{f}\left(G_{[1,1]}\right) \leqslant 4.36$. Note that the upper bound is much smaller than the upper bound for $\chi\left(G_{[1,1]}\right)$.

\subsection{Practical Motivation}

Coloring of such geometrical graphs has also some significant practical motivations in telecommunication. We will briefly describe an example. More on this topic can be found in the paper of Walczak and Wojciechowski [11].

Consider the following problem: We are given a set of transmitters with equal ranges placed in some area (assume that ranges are equal to 1) - some of them are in each other range, and some of them are not. If two transmitters are in each other's range, we assume that they can 'quickly' agree on their communication (there are algorithms for it). If two transmitters are not in each other's range but have a common neighbor $C$, then it is possible that $A$ and $B$ would try to transmit to $C$ in the same time-in this case $C$ cannot listen to both messages. Hence we have a conflict which cannot be solved by a direct communication between $A$ and $B$. If two transmitters $A$ and $B$ are not in each other's range and do not have a common neighbor, then there is no conflict. The problem is to assign time-slots for transmitters (in an equitable way) such that no two conflicted transmitters share a time interval.

How can we use graph coloring in this problem? We can create the graph $G$ of conflicts for this network of transmitters: vertices correspond to transmitters and two vertices are adjacent if the corresponding transmitters $X, Y$ are at distance greater than 1 and have a common transmitter in their respective ranges (note that it is possible only if $X$ and $Y$ are at distance in the set $(1,2)$ ). Hence, using a $k$-coloring of $G$, such that each color corresponds to one time-slot is one of the possible ways of constructing a proper transmission-schedule. By the definition of coloring, the produced schedule does not contain any pair of conflicted transmitters sharing their time of transmission and every transmitter gets the same amount of time in one cycle of transmission. The length of the schedule is $k$. On the other hand, we can make use of a fractional coloring of $G$. Since we demand that in one cycle of transmission every transmitter gets a unit of time, then every color in a fractional coloring corresponds to an interval of length $1 / j$ of the time unit. This model guarantees avoiding of conflicts as well as 
equability of the schedule (if we used $k$ colors then the length of the schedule is $k / j$ ). The relation between considered coloring models implies that fractional coloring can possibly produce a shorter schedule in comparison to classic coloring, which (in real world) means: we can save a bit of time. However, the technical constraints imply that too big values of $j$ are not accepted-too fragmented schedule is not practical. This issue plays an important role in our work-we devote a part of this article to fractional colorings with 'small $j$ '.

Now, consider a graph on the set of vertices $\mathbb{R}^{2}$ with vertices at distance in the set $[1,2]$ adjacent, which will be denoted in this article as $G_{[1,2]}$. Clearly, every conflict graph of a transmitters network as described above is a subgraph of $G_{[1,2]}$. Therefore, any coloring or fractional coloring of infinite $G_{[1,2]}$ induce, respectively, a coloring or a fractional coloring of a given finite conflict graph. Hence, we get a universal scheme for any network. Additionally, this universal scheme works also if the transmitters are placed on moving vehicles (thus changing the conflict graph). This is an indisputable advantage over using standard coloring algorithms for a given conflict graph.

\section{Preliminaries}

First, we need to formally define fractional and classic coloring of graphs.

Definition 1 A coloring of a graph $G=(V, E)$ with $k$ colors (or $k$-coloring) is an assignment of colors $\{1,2, \ldots, k\}$ to the vertices of $G$ such that no two adjacent vertices have the same color. The smallest number of colors needed to color a graph $G$ is called chromatic number and denoted by $\chi(G)$.

Definition 2 Let $P_{j}(k)$ be a family of all $j$-elemental subsets of $\{1,2, \ldots, k\}$. A $j$ fold coloring of graph $G=(V, E)$ is an assignment of $j$-element sets of colors to the vertices of $G$, i.e. $f: V \longrightarrow P_{j}(k)$, such that for any two adjacent vertices $v, w \in V$ we have $f(v) \cap f(w)=\emptyset$. The smallest number of colors $k$ needed for a $j$-fold coloring of a graph $G$ is called the $j$-fold chromatic number and denoted by $\chi_{j}(G)$. The fractional chromatic number is defined to be

$$
\chi_{f}(G):=\inf _{j \in \mathbb{N}} \frac{\chi_{j}(G)}{j}=\lim _{j \rightarrow \infty} \frac{\chi_{j}(G)}{j} .
$$

Since a 1-fold coloring of a graph is just a classic coloring $\chi_{f}(G) \leqslant \chi(G)$.

Now, we will present two possible, equivalent notions of graphs on the Euclidean plane we are considering in this work. The first is due to Exoo [1]. The second is introduced in this paper and is more convenient for our work, except Sect. 3.

Definition 3 An $\varepsilon$-unit distance graph, denoted by $G_{\varepsilon}$ is a graph whose vertices are all points of the plane, in which two points are adjacent if their distance $d$ satisfies $1-\varepsilon \leqslant d \leqslant 1+\varepsilon$, i.e.

$$
G_{\varepsilon}=\left(\mathbb{R}^{2},\left\{\{x, y\} \subset \mathbb{R}^{2} \mid 1-\varepsilon \leqslant \operatorname{dist}(x, y) \leqslant 1+\varepsilon\right\}\right) .
$$


Definition 4 A graph $G_{[a, b]}$ is a graph whose vertices are all points of the plane $V=\mathbb{R}^{2}$, in which two points are adjacent if their distance $d$ satisfies $a \leqslant d \leqslant b$.

$$
G_{[a, b]}=\left(\mathbb{R}^{2},\left\{\{x, y\} \subset \mathbb{R}^{2} \mid a \leqslant \operatorname{dist}(x, y) \leqslant b\right\}\right) .
$$

Note that every $G_{\varepsilon}$ graph can be defined as $G_{[1-\varepsilon, 1+\varepsilon]}$ and every $G_{[a, b]}$ graph can be defined as $G_{\varepsilon}$ graph with $\varepsilon=\frac{b-a}{b+a}$. Additionally, it is enough to consider graphs $G_{[1, b]}$ since $G_{[a, b]} \cong G_{[1, b / a]}$.

\section{Coloring of $G_{[a, b]}$}

The classic Hadwiger-Nelson problem is considered to be very difficult, in particular giving a better general lower bound than 4. In his article Exoo [1] looked for values of $\varepsilon$ such that we can determine the chromatic number of $G_{\varepsilon}$ and he succeed in finding some such values. He proved that for $0.134756 \ldots<\varepsilon<0.138998 \ldots$ the exact value of $\chi\left(G_{\varepsilon}\right)$ is 7 , and for $\varepsilon>0.008533 \ldots$ we get $\chi\left(G_{\varepsilon}\right) \geqslant 5$.

His work (including computational experiments) suggested that for small enough $\varepsilon$ the exact value of the chromatic number of $G_{\varepsilon}$ is 7 .

Conjecture 1 [1] For any $\varepsilon>0$ we have $\chi\left(G_{\varepsilon}\right)=7$.

We will give a partial answer to this conjecture. It appears that the pure positivity of $\varepsilon$ allow us to establish a lower bound of 5, strictly better than 4 . We will use a result by Nielsen [8] and for that we need some additional notions.

Given a coloring of the plane $F$, a triangle $T=x y z$ is a monochromatic limit triangle if there is a monochromatic set $\left\{x_{1}, y_{1}, z_{1}, x_{2}, y_{2}, z_{2}, \ldots\right\}$ such that $x_{n} \longrightarrow x$, $y_{n} \longrightarrow y, z_{n} \longrightarrow z$ and each of the triangles $T_{n}=x_{n} y_{n} z_{n}$ is similar to $T$.

We will say that triangles $x y z$ and $x^{\prime} y^{\prime} z^{\prime}$ are $\varepsilon$-close if $\left\|x-x^{\prime}\right\|<\varepsilon,\left\|y-y^{\prime}\right\|<\varepsilon$ and $\left\|z-z^{\prime}\right\|<\varepsilon$.

Theorem 1 [8] Let $F$ be a two-coloring of the plane and let $T$ be a triangle. Then $F$ admits a monochromatic limit triangle congruent to $T$.

Theorem 2 For any $\varepsilon>0$ we have $\chi\left(G_{\varepsilon}\right) \geqslant 5$.

Proof Let $\varepsilon>0$ and suppose that $\chi\left(G_{\varepsilon}\right) \leqslant 4$. Let $F$ be a 4-coloring of $G_{\varepsilon}$ and let $F^{\prime}$ be a 2-coloring of the plane such that each point of color 1 or 2 in $F$ is white in $F^{\prime}$ and each point of color 3 or 4 in $F$ is black in $F^{\prime}$. Let $T=x y z$ be an equilateral triangle with a side length 1 .

From Theorem $1 F^{\prime}$ admits a monochromatic limit triangle congruent to $T$ (lets say it is white). So from the definition of monochromatic limit triangle there exists $n$ such that the triangle $T_{n}=x_{n} y_{n} z_{n}$ is $\frac{\varepsilon}{2}$-close to $T$. The side lengths of $T_{n}$ are within the interval $[1-\varepsilon, 1+\varepsilon]$, hence the sides of $T_{n}$ are edges of $G_{\varepsilon}$. Since all vertices of $T_{n}$ are white in $F^{\prime}$ then each of them is colored with 1 or 2 in $F$. Since there are three vertices and two colors there is a monochromatic edge, which contradicts the assumption that $F$ is a 4-coloring of $G_{\varepsilon}$.

We believe that there is still room to prove a better bound. 


\section{Fractional Chromatic Number of $G_{[a, b]}$}

The best known way to find an upper bound for the fractional chromatic number of $G_{[1,1]}$ is by looking for a set in the plane with highest possible density but without vertices in distance equal to 1 . The densest such set was presented by Hochbeg and O'Donnell [5]. They presented a proof of the upper bound by a limit argument. In the following theorem we generalize their construction for $G_{[1, b]}$. We give a complex description of the method for any $b$ : not only the upper bound, but also an explicit specification of a sequence of fractional colorings "converging" to the upper bound. This sequence give a finite fractional coloring with "quality" as close to the upper bound as needed.

Theorem 3 If $b \geq 1$ then $\chi_{f}\left(G_{[1, b]}\right) \leqslant \frac{\sqrt{3}}{3} \cdot \frac{b+\sqrt{1-x^{2}}}{x}$ where $x$ is the root of $b x=\frac{\pi}{6}-\operatorname{arc} \sin (x)$. Moreover there exists a sequence of $\left(\frac{n}{2(b+1)}-1\right)^{2}$-fold colorings with $n^{2}$ colors for $n \geqslant 1$.

Proof Note: In order to keep this proof shorter we omit some details of calculations. We will define a set $S$ in the plane with high density but without vertices in distance in $[1, b]$ in the following way. Let set $A$ be an intersection of a disk of unit diameter and a hexagon with common center point as in Fig. 1. The shape of $A$ changes when we change the size of the hexagon. In the fractional coloring of $G_{[1, b]}$ we choose the set $A$ in such a way that the ratio between the length of the circular arc $y$ and the segment $x$ is equal to $b$ (it will be explained later).

From the construction we get the following equalities: $x=\sin \left(\frac{\alpha}{2}\right), y=\frac{\beta}{2}$, $\alpha+\beta=\frac{\pi}{3}$. Therefore we obtain

$$
y=\frac{\pi}{6}-\arcsin (x) .
$$

Then we build $S$ by placing copies of $A$ on the plane with distance $b$ like in Fig. 2 . Assume that two neighboring components of $S$ are centered at $(0,0)$ and $(s, 0)$ which determines the location of all components of $S$. Let $n$ be a positive integer. We define a tiling with hexagons of width $s / n$ in such a way that the left side of some hexagon is a part of the left vertical segment of the border of $A$.

For $0 \leqslant i, j<n$ let $S_{i, j}=S+(s i / n)(1,0)+(s j / n)(1 / 2, \sqrt{3} / 2)$. We define $h_{n}$-fold coloring of the plane as follows. We assign color $(i, j)$ to all points in hexagons
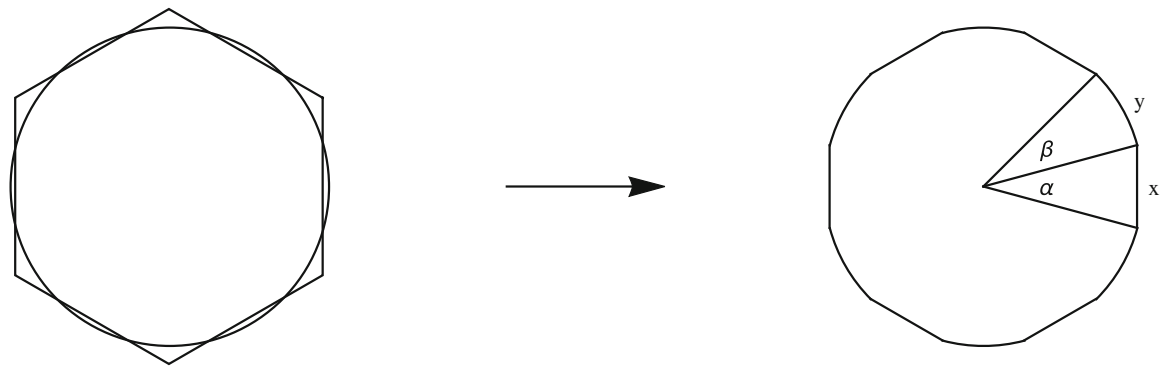

Fig. 1 Set $A$ 


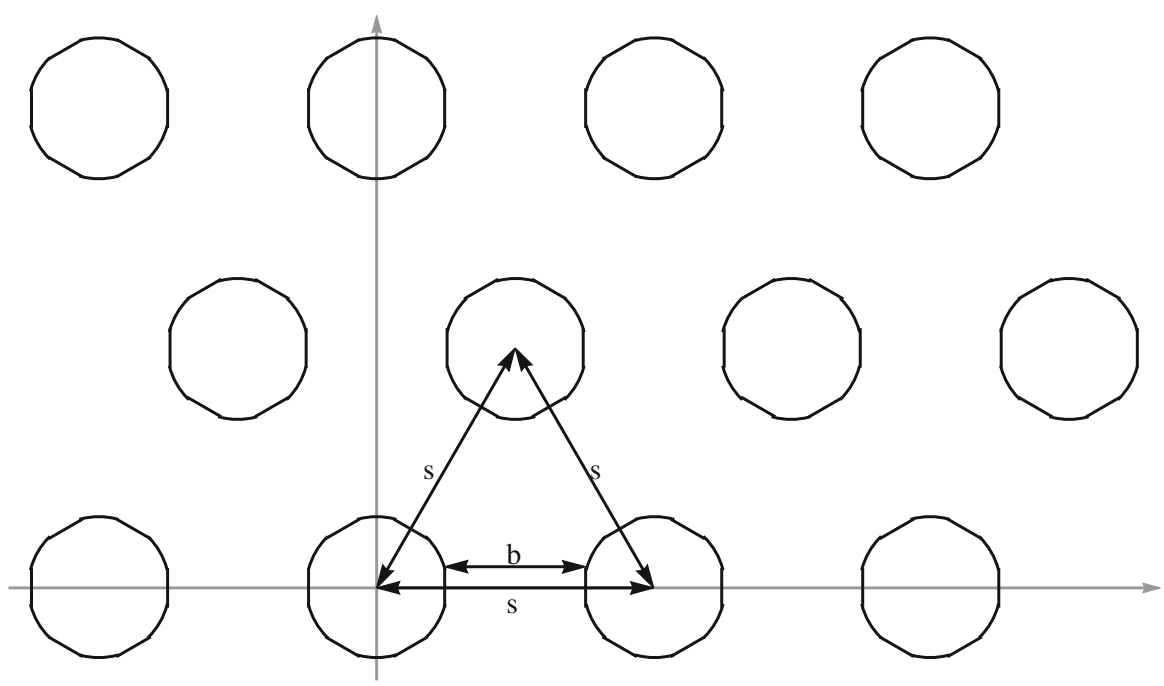

Fig. 2 Set $S$

fully contained in $S_{i, j}$. Now we will estimate the number $h_{n}$ of hexagons that are fully contained in one copy of $A$, let say $A^{0}$. Let $A_{n}^{0}$ be a rescaled copy o $A$ with the same center as $A^{0}$ and diameter $\left(1-2 \frac{s}{n}\right)$. The area of $A_{n}^{0}$ is $p_{n}=\frac{1}{4}\left(1-2 \frac{s}{n}\right)^{2}$ $\left(\pi-6 \arcsin (x)+6 x \sqrt{1-x^{2}}\right)$, and the area of the hexagon is $(s / n)^{2}(\sqrt{3} / 2)$. Observe that if a hexagon of the tiling has non-empty intersection with $A_{n}^{0}$ then it is fully contained in $A^{0}$. Therefore we can bound $h_{n}$ from below:

$$
\begin{aligned}
h_{n} & \geqslant \frac{p_{n}}{(s / n)^{2}(\sqrt{3} / 2)}=\frac{\frac{1}{4}\left(1-2 \frac{s}{n}\right)^{2}\left(\pi-6 \arcsin (x)+6 x \sqrt{1-x^{2}}\right)}{(s / n)^{2}(\sqrt{3} / 2)} \\
& =\sqrt{3}\left(\frac{n}{b+\sqrt{1-x^{2}}}-2\right)^{2}\left(b x+x \sqrt{1-x^{2}}\right)
\end{aligned}
$$

On the other hand we conclude that every hexagon is contained in $h_{n}$ of $S_{i, j}$ (since we can get all the hexagons by shifting one of them by $(s i / n)(1,0)+(s j / n)(1 / 2, \sqrt{3} / 2))$, hence every point in a hexagon has $h_{n}$ of $n^{2}$ colors assigned.

With $n$ tending to infinity the set of points colored 1 tends to $S$ and our sequence of $h_{n}$-fold colorings gives us an upper bound for the fractional chromatic number of $G_{[1, b]}$ :

$$
\begin{aligned}
\chi_{f}\left(G_{[1, b]}\right) & \leqslant \lim _{n \rightarrow \infty} \frac{n^{2}}{\frac{1}{2 \sqrt{3}}\left(\frac{n}{b+\sqrt{1-x^{2}}}-2\right)^{2}\left(\pi-6 \arcsin (x)+6 x \sqrt{1-x^{2}}\right)} \\
& =\frac{2 \sqrt{3}}{\pi-6 \arcsin (x)+6 x \sqrt{1-x^{2}}} \cdot \lim _{n \rightarrow \infty} \frac{n^{2}}{\left(\frac{n}{b+\sqrt{1-x^{2}}}-2\right)^{2}}
\end{aligned}
$$




$$
\begin{aligned}
& =\frac{2 \sqrt{3}\left(b+\sqrt{1-x^{2}}\right)^{2}}{\pi-6 \arcsin (x)+6 x \sqrt{1-x^{2}}} \\
& =\frac{2 \sqrt{3}\left(b+\sqrt{1-x^{2}}\right)^{2}}{\pi-6 \arcsin (x)+3 \sin (2 \arcsin (x))} .
\end{aligned}
$$

Now let us explain why did we choose the ratio between the length of the circular arc $-y$ and the segment $-x$ to be $b$. To find minimal value we take the derivative of the upper bound for $\chi_{f}\left(G_{[1, b]}\right)$ presented above and check when it equals 0 .

$$
\begin{aligned}
& \frac{4 \sqrt{3} x\left(b+\sqrt{1-x^{2}}\right)(6 b x+6 \arcsin (x)-\pi)}{\sqrt{1-x^{2}}(-6 \arcsin (x)+3 \sin (2 \arcsin (x))+\pi)^{2}}=0 \\
& \Longleftrightarrow 6 b x+6 \arcsin (x)-\pi=0 .
\end{aligned}
$$

Applying equality 1 we obtain

$$
6 b x-6 y=0
$$

and hence

$$
y=b x .
$$

To complete the proof we transform the formula

$$
\frac{2 \sqrt{3}\left(b+\sqrt{1-x^{2}}\right)^{2}}{\pi-6 \arcsin (x)+3 \sin (2 \arcsin (x))}
$$

using the previous equality and by doing so we get

$$
\frac{\sqrt{3}}{3} \cdot \frac{b+\sqrt{1-x^{2}}}{x}
$$

as the upper bound for the fractional chromatic number of graph $G_{[1, b]}$.

Figure 3 and Table 1 present the upper bound for the fractional chromatic number of $G_{[1, b]}$ for different values $b$.

\section{$5 j$-Fold Coloring of the Plane}

Note that the upper bounds for the fractional chromatic numbers of $G_{[1, b]}$ are established by presenting an infinite sequence of $j$-fold colorings. These colorings give results close to the upper bound only for very big $j$. However, as it was mentioned in the introduction, for the practical reasons it is often the case that only $j$-fold coloring with small $j$ are valuable to consider. Furthermore, this consideration leads also to a purely mathematical question: How fast, in terms of $j$, can we get close to the infinite limit - the upper bound for the fractional chromatic number? In this section, we give some insight into this matter. 


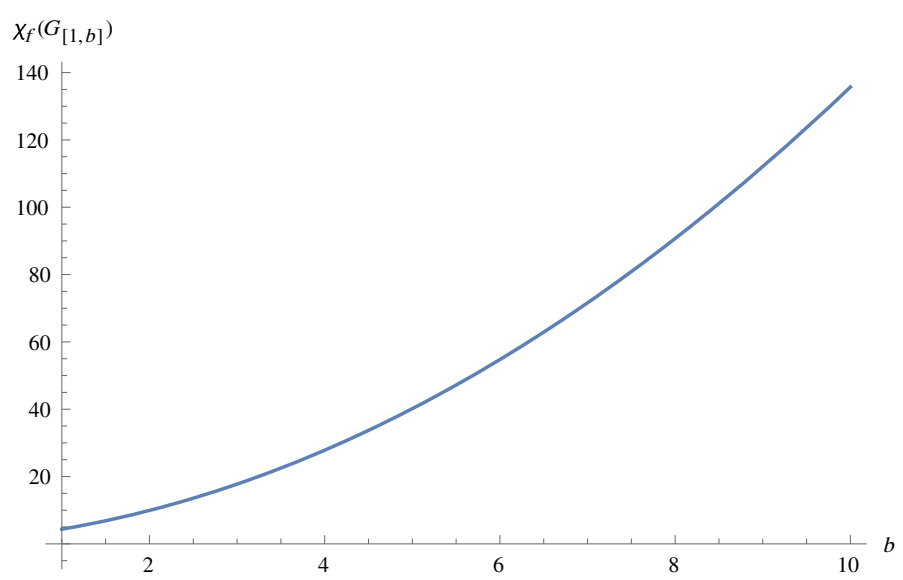

Fig. 3 The upper bound for $\chi_{f}\left(G_{[1, b]}\right)$ from Theorem 3

Table 1 Applications of Theorem 3

\begin{tabular}{llllll}
\hline$b=$ & 1 & 1.5 & 2 & 3 & 4 \\
\hline$\chi_{f}\left(G_{[1, b]}\right) \leqslant$ & 4.36 & 6.86 & 9.9 & 17.62 & 27.55 \\
\hline
\end{tabular}

\subsection{Methods for $G_{[1,1]}$}

In this subsection, we concentrate on $G_{[1,1]}$.

Theorem 4 There are twofold coloring of $G_{[1,1]}$ with 12 colors and threefold coloring of $G_{[1,1]}$ with 16 colors.

Proof For our colorings we are going to use classic covering of the plane with hexagons with side length $1 / 2$. Obviously in a hexagon of side length $1 / 2$ there are pairs of point in a distance 1 , so when we say we color a hexagon we mean the interior of the hexagon plus its right border and its three vertices: the upper one and the two on the right (see Fig. 4).

Firstly we show our twofold coloring of $G_{[1,1]}$ with 12 colors. We are going to use the hexagon grid twice with the set of colors $\{1,2, \ldots, 12\}$. We create the first layer by simply giving each row of hexagons three numbers and use it periodically so that if one row has colors from the set $\{1,2,3\}$ the next gets colors $\{4,5,6\}$ and so on. Having this coloring as our model we get the second layer by moving the colored grid by a vector $[3 \sqrt{3} / 4,-3 / 2]$. See Fig. 5 with the first layer of colors and marked placement of color 1 in the layers. The distances between two hexagons of the same color are: vertically 2 , horizontally $\sqrt{3}$ and diagonally $\frac{5 \sqrt{3}}{8} \approx 1.08$, so they are all bigger than 1.

To create a threefold coloring of $G_{[1,1]}$ with 16 colors we again start with the classic covering of the plane with hexagons with side length $1 / 2$. We are going to use the hexagon grid 3 times with the set of colors $\{1,2,3, \ldots, 16\}$. We create the first layer by simply giving each row of hexagons four numbers and use it periodically so 
Fig. 4 Borders of a hexagon

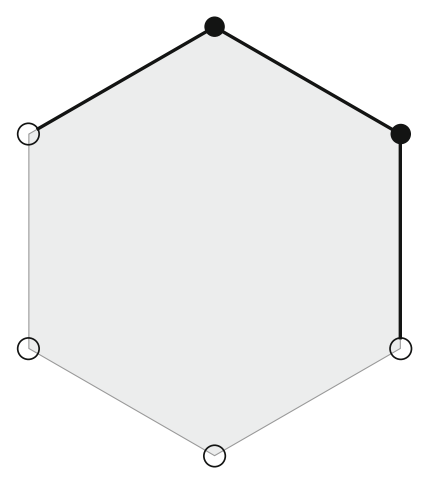

Fig. 5 Twofold coloring of $G_{[1,1]}$ with 12 colors

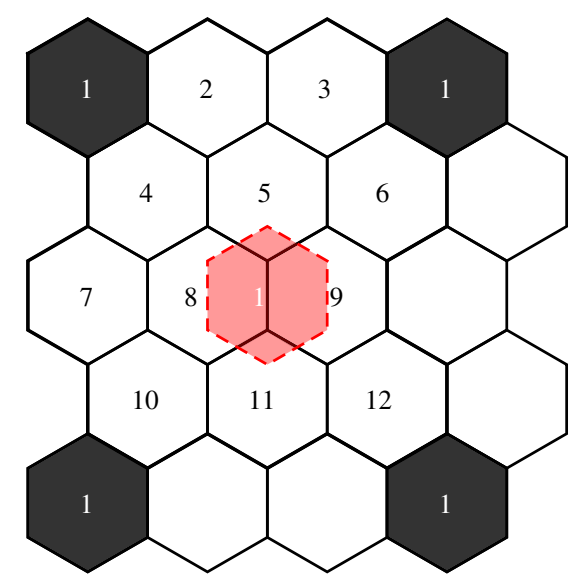

that if one row has colors from the set $\{1,2,3,4\}$ the next gets colors $\{5,6,7,8\}$ and so on. Having this coloring as our model we get second and third layers by moving the colored grid by a vector $[\sqrt{3},-1]$. See Fig. 6 with the first layer of colors and marked placement of color 1 in other layers. The distances between two hexagons of the same color are at least one (exactly one in case of two hexagons from different layers).

Theorem 5 There is a sevenfold coloring of $G_{[1,1]}$ with 37 colors i.e. $\frac{37}{7} \approx 5.285$.

Proof To create sevenfold coloring of $G_{[1,1]}$ we start with a hexagon grid with hexagon side's length $s=\frac{1}{2 \sqrt{7}}$. We color with the first color some hexagons in the pattern presented as shadowed on Fig. 7. Then we shift our pattern by a vector $\left[\frac{\sqrt{3}}{2 \sqrt{7}}, 0\right]$ and color it with second color (bold contour in Fig. 7). Repeating this action 37 times we have 7 colors for each hexagon and there are 37 colors total. In our base coloring figure made by 7 hexagons the largest distance between two points is 1 so choosing half of the border to be colored and the other not is enough to make sure there are no two point in distance 1 in one figure. The distance between two such figures is 


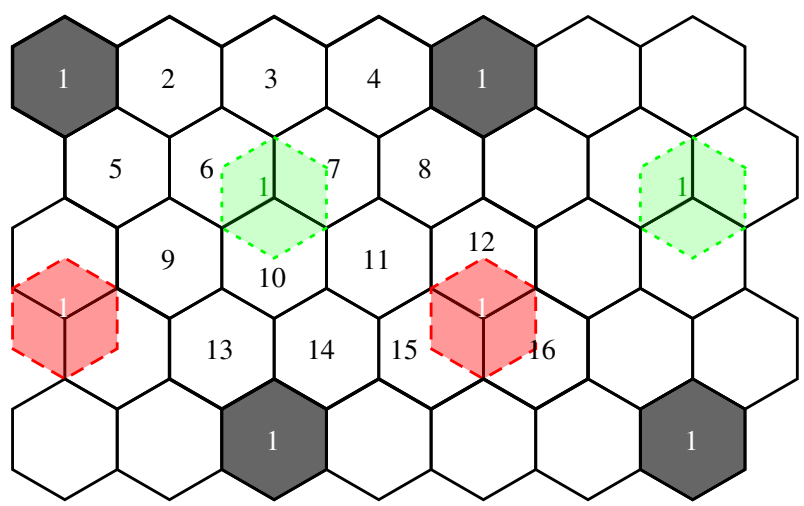

Fig. 6 Threefold coloring of $G_{[1,1]}$ with 16 colors

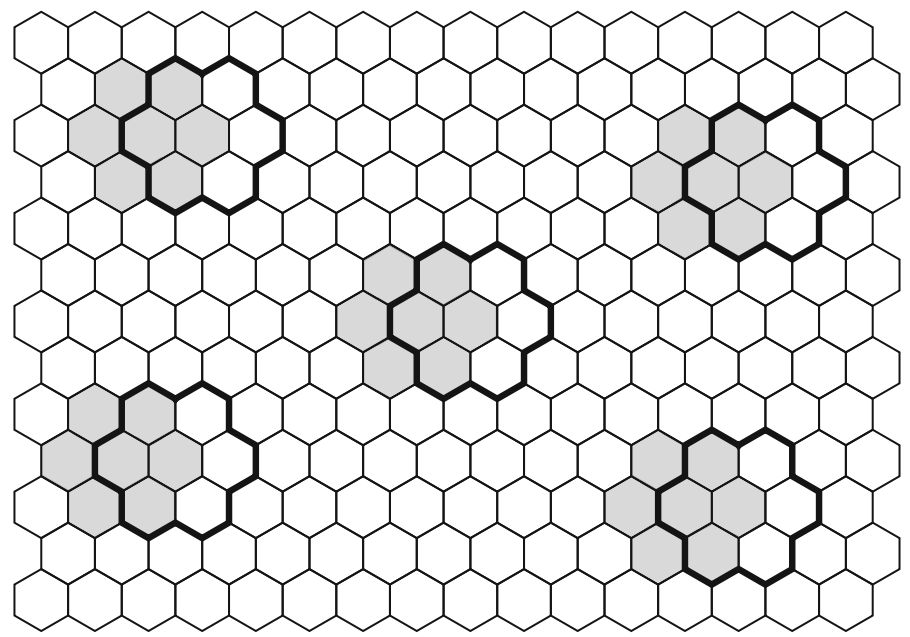

Fig. 7 Sevenfold coloring of $G_{[1,1]}$ with 37 colors

$$
\sqrt{(3 \sqrt{3} s)^{2}+(2 s)^{2}}=s \sqrt{31}=\frac{\sqrt{31}}{2 \sqrt{7}} \approx 1.05
$$

\subsection{Methods for $G_{[a, b]}$}

In this section we give two general methods for building $j$-fold colorings (for small $j$ ) for graphs $G_{[1, b]}$.

Theorem 6 There exists a nm-fold coloring with $\left\lceil\left(\frac{2 b}{\sqrt{3}}+1\right) \cdot n\right\rceil \cdot\left\lceil\left(\frac{2 b}{\sqrt{3}}+1\right) \cdot m\right\rceil$ colors of the graph $G_{[1, b]}$ i.e.

$$
\frac{\chi_{n m}\left(G_{[1, b]}\right)}{n m} \leqslant \frac{\lceil(2 b / \sqrt{3}+1) \cdot n\rceil \cdot\lceil(2 b / \sqrt{3}+1) \cdot m\rceil}{n m} .
$$




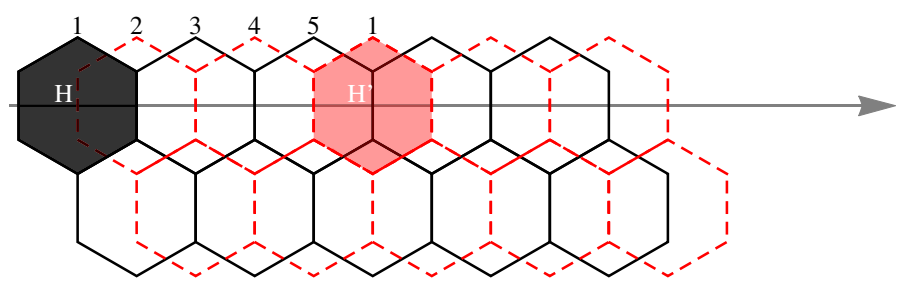

Fig. 8 Twofolds in fourfold coloring $G_{[1,1]}$ with 25 colors (different shades/colors represent color $(1,1)$ from different layers)

Proof In the proof we are going to create $n \cdot m$ colored hexagon grids. A color of a hexagon will be a pair of numbers, first of which will be related to the row the hexagon is in and the second corresponds to the column.

Let $W_{1}^{1}$ be a hexagon grid with hexagons with side length $1 / 2$. Let $H$ be one of the hexagons from $W_{1}^{1}$. For $2 \leqslant j \leqslant n$ let $W_{1}^{j}$ be a hexagon grid created by moving uncolored $W_{1}^{1}$ by a vector $(j-1) / n[\sqrt{3} / 2,0]$. Now, lets say $H$ is colored $(1,1)$ and let's find first hexagon $H^{\prime}$ in the same row from any $W_{1}^{j}$ that can also be colored $(1,1)$ without creating a monochromatic edge in $G_{[1, b]}$ i.e at distance greater or equal to $b$ (see Fig. 8).

Counting the hexagons from $H$ to $H^{\prime}$ we find that there are exactly

$$
\left\lceil\left(b+\frac{\sqrt{3}}{2}\right) \frac{2 n}{\sqrt{3}}\right\rceil=\left\lceil\left(\frac{2 b}{\sqrt{3}}+1\right) \cdot n\right\rceil
$$

of them, since the distance between the centers of $H$ and $H^{\prime}$ has to be at least $b+\frac{\sqrt{3}}{2}$. Then we shift each of $W_{1}^{j}$ by vectors $(i-1) / m[\sqrt{3} / 4,-3 / 4]$ for $2 \leqslant i \leqslant m$ getting grids $W_{i}^{j}$. Now remembering that $H$ is colored $(1,1)$ we find the first hexagon $H^{\prime \prime}$ from $W_{i}^{1}$ to have the same color (see Fig. 9). The number of hexagons between the two is $\lceil(2 b / \sqrt{3}+1) \cdot m\rceil$.

Now we have three hexagons $H, H^{\prime}$, and $H^{\prime \prime}$ that can be colored with the same color, since the distance between $H^{\prime}$ and $H^{\prime \prime}$ would be the smallest when $n=m$ and then the centers of the three hexagons create an equilateral triangle with side length at least $b+\frac{\sqrt{3}}{2}$ (because $H$ and $H^{\prime}$ are in proper distance).

The final coloring is created by giving each hexagon a pair of numbers, so that $H$ gets color $(1,1)$, the next hexagon in the same row gets $(1,2)$ and so on until $H^{\prime}$ gets $(1,1)$ again and the cycle repeats, and in the row below we get colors $(2,1),(2,2),(2,3) \ldots$ and so on until we have row with $H^{\prime \prime}$ in which we use $(1,1),(1,2),(1,3) \ldots$ again. Since we have $n m$ hexagon grids every point gets $n m$ colors. Finally we get $n m$-fold coloring of $G_{[1, b]}$ with $\left\lceil\left(\frac{2 b}{\sqrt{3}}+1\right) \cdot n\right\rceil \cdot\left\lceil\left(\frac{2 b}{\sqrt{3}}+1\right) \cdot m\right\rceil$ colors (Fig. 10).

The following result can be seen as a combination of twofold coloring approach from Theorem 4 and the main method from Theorem 6 . 


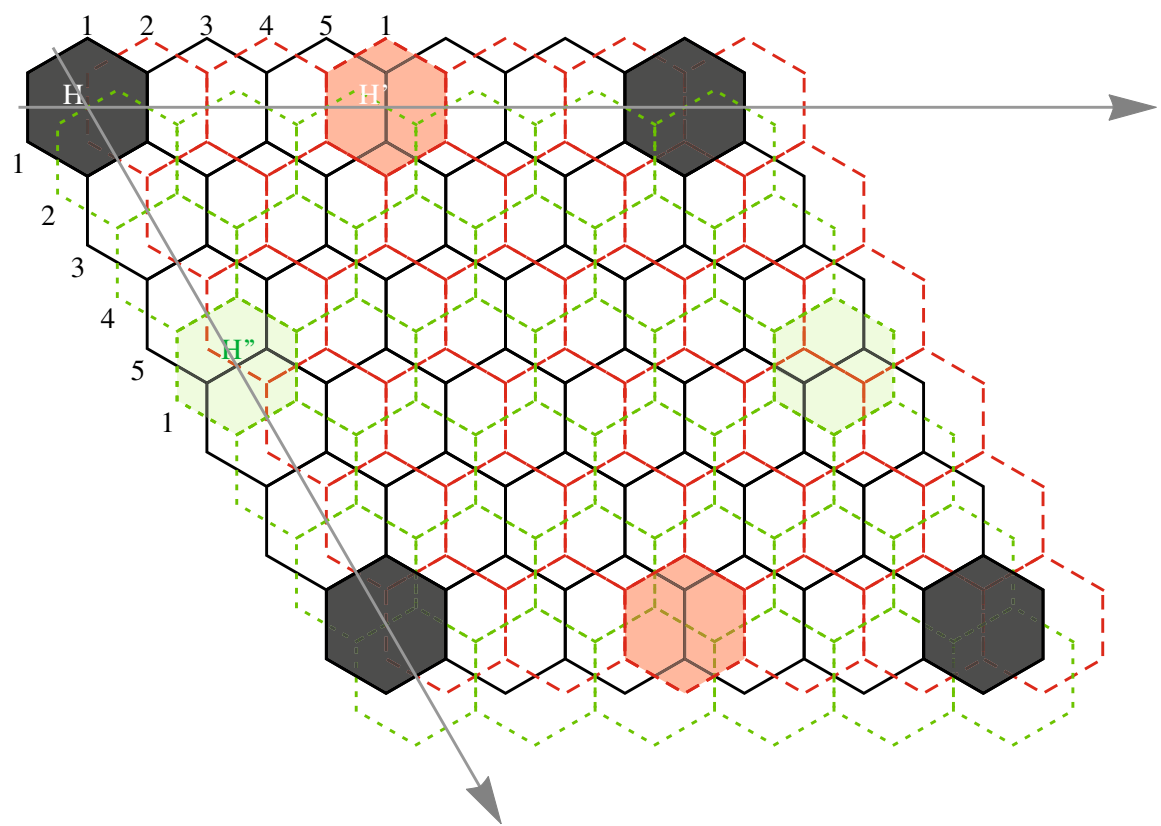

Fig. 9 Threefolds in fourfold coloring $G_{[1,1]}$ with 25 colors (different shades/colors represent color $(1,1)$ from different layers)

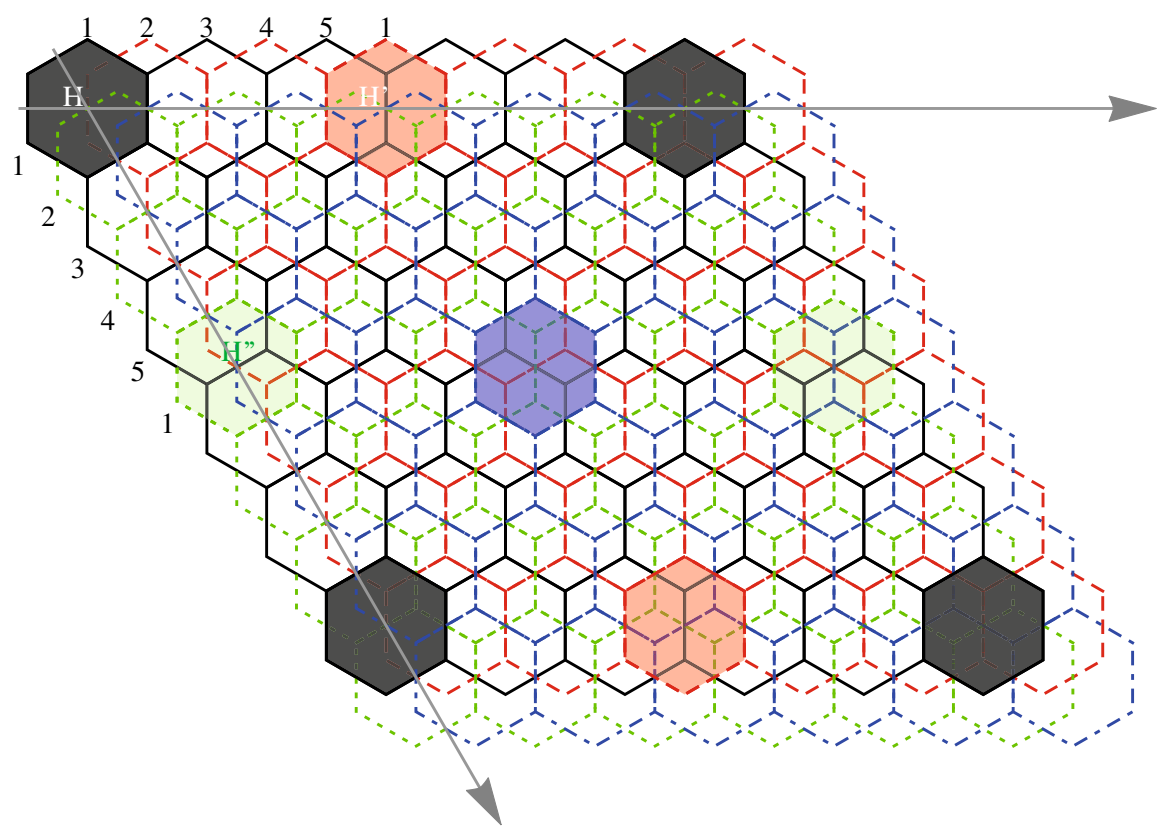

Fig. 10 Fourfold coloring $G_{[1,1]}$ with 25 colors (different shades/colors represent color $(1,1)$ from different layers) 
Theorem 7 There exists a $2 n m$-fold coloring with $2\lceil(b+1) 2 n\rceil\left\lceil(b+1) \frac{2 m}{3}\right\rceil$ colors of the graph $G_{[1, b]}$ i.e.

$$
\frac{\chi_{2 n m}\left(G_{[1, b]}\right)}{2 n m} \leqslant \frac{2\left\lceil\sqrt{3}(b+1) \frac{2 n}{3}\right\rceil\left\lceil(b+1) \frac{2 m}{3}\right\rceil}{2 n m} .
$$

Proof Note that the proof uses similar methods to the previous one. We are going to create $2 \cdot n \cdot m$ colored hexagon grids. A color of a hexagon will be a pair of numbers, first of which will be related to the row the hexagon is in and the second corresponds to the column.

Let $W_{1}^{1}$ be a hexagon grid with hexagons with side length $1 / 2$. Let $H$ be one of the hexagons from $W_{1}^{1}$. For $2 \leqslant i \leqslant m$ let $W_{i}^{1}$ be a hexagon grid created by moving uncolored $W_{1}^{1}$ by a vector $(i-1) / m[0,-3 / 2]$. Now lets say $H$ is colored $(1,1)$ and let's find first hexagon $H^{\prime}$ in the same column from any $W_{i}^{1}$ that can also be colored $(1,1)$ without creating a monochromatic edge in $G_{[1, b]}$ i.e at distance greater or equal to $b$, so the distance between the centers of $H$ and $H^{\prime}$ needs to be at least $b+1$ (see Fig. 11 for fourfold coloring with $4 \cdot 6=24$ colors of $G_{[1,1]}$ with $n=1$ and $m=2$ )

Since the distance between the centers of $H$ and $H^{\prime}$ is at least $b+1$ but we chose $H^{\prime}$ to be as close as possible to $H$, then there are $\left\lceil(b+1) \frac{2 m}{3}\right\rceil$ rows between $H$ and $H^{\prime}$. Now for $2 \leqslant j \leqslant m$ and $2 \leqslant j \leqslant n$ let $W_{i}^{j}$ be a hexagon grid created by shifting $W_{i}^{1}$ by a vector $(j-1) / n[\sqrt{3} / 2,0]$. Let $H^{\prime \prime}$ be the first hexagon in the same row as $H$ in any of $W_{1}^{j}$ such that the distance between the centers of the two is at least $\sqrt{3}(b+1)$. The number of hexagons between $H$ and $H^{\prime \prime}$ is $\left\lceil\sqrt{3}(b+1) \frac{2 n}{\sqrt{3}}\right\rceil=\lceil(b+1) 2 n\rceil \geqslant b+\frac{\sqrt{3}}{2}$.

The distance between the centers of $H^{\prime}$ and $H^{\prime \prime}$ is at least:

$$
\begin{aligned}
& \sqrt{\left(\left\lceil(b+1) \frac{2 m}{3}\right\rceil \frac{3}{2 m}\right)^{2} \cdot\left(\left\lceil\sqrt{3}(b+1) \frac{2 n}{\sqrt{3}}\right\rceil \frac{\sqrt{3}}{2 n}\right)^{2}} \\
& \geqslant \sqrt{(b+1)^{2}+(\sqrt{3}(b+1))^{2}}=\sqrt{4(b+1)^{2}}=2 b+2 .
\end{aligned}
$$

Since the distance is at least $2 b+2$ they can be colored the same color and there is enough space between them to put another hexagon in the same color between them. So we create new hexagon grids $V_{i}^{j}$ by shifting $W_{i}^{j}$ by a vector

$$
\left\lceil\left\lceil\sqrt{3}(b+1) \frac{2 n}{\sqrt{3}}\right\rceil \cdot \frac{\sqrt{3}}{4 n},\left\lceil(b+1) \frac{2 m}{3}\right\rceil \cdot \frac{3}{4 m}\right] .
$$

The final coloring is created by giving each hexagon a pair of numbers, so that $H$ gets color $(1,1)$ the next hexagon in the same row from any $W_{1}^{j}$ gets $(1,2)$ and so on until $H^{\prime \prime}$ gets $(1,1)$ again and the cycle repeats. The next row from $W_{1}^{j}$ has the first coordinate in all colors equal to 2 . The set of numbers for second coordinate is $\left\{1,2, \ldots,\left\lceil\sqrt{3}(b+1) \frac{2 n}{\sqrt{3}}\right\rceil\right\}$, and for the first coordinate $2\left\lceil(b+1) \frac{2 m}{3}\right\rceil$ (since there are 2 rows of hexagons between 2 hexagons from the same grid in the same column). So we use $2\left\lceil(b+1) \frac{2 m}{3}\right\rceil \cdot\left\lceil\sqrt{3}(b+1) \frac{2 n}{\sqrt{3}}\right\rceil$ colors using $2 n m$ hexagon grids. 
Fig. 11 Fourfold coloring $G_{[1,1]}$ with 24 colors (different shades/colors represent color $(1,1)$ from different layers)
Table 2 Applications of Theorems 6 and 7 for $G_{[1,1]}$ with small $j$

Table 3 Selected results in $j$-fold coloring of $G_{[1,1]}$ with small $j$

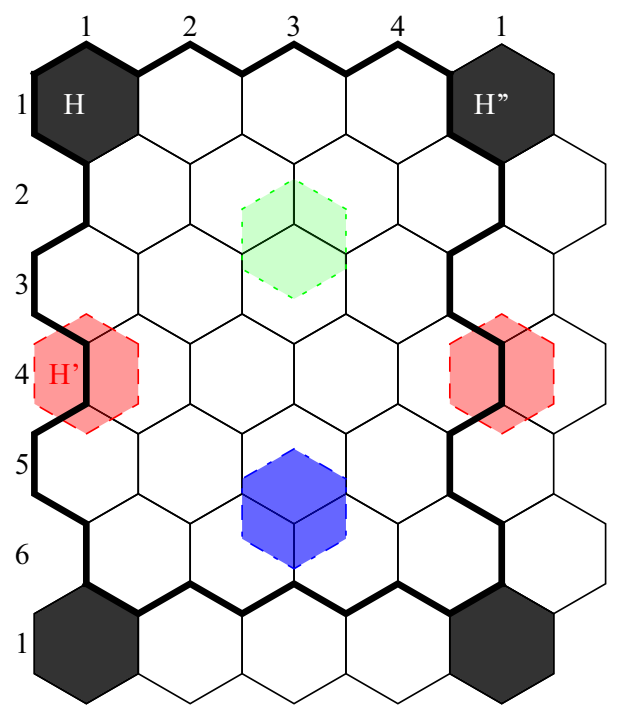

\begin{tabular}{llllllll}
\hline Method & $j=$ & 2 & 4 & 6 & 8 & 10 & 12 \\
\hline$j=\mathrm{nm}$ & $k=$ & $\mathbf{1 5}$ & 25 & 35 & $\mathbf{4 5}$ & $\mathbf{5 5}$ & $\mathbf{6 3}$ \\
& $k / j \approx$ & 7.5 & 6.25 & 5.83 & 5.63 & 5.5 & 5.25 \\
$j=2 \mathrm{~nm}$ & $k=$ & 16 & $\mathbf{2 4}$ & $\mathbf{3 2}$ & 48 & 56 & 64 \\
& $k / j \approx$ & 8 & 6 & 5.33 & 6 & 5.6 & 5,33
\end{tabular}

\begin{tabular}{llllllll}
\hline$k=$ & 7 & 12 & 16 & 24 & 32 & 32 & 37 \\
\hline$j=$ & 1 & 2 & 3 & 4 & 5 & 6 & 7 \\
$k / j \approx$ & 7 & 6 & 5.33 & 6 & 6.6 & 5.33 & 5.26
\end{tabular}

\subsection{Summary}

Theorems 6 and 7 give different results and we cannot say one is stronger than another. Table 2 presents comparison of the two for $j$-fold coloring of $G_{[1,1]}$ with small values of $j$ ( $k$ is the numbers of colors used). We bold best results for a fixed $j$.

Our best results in $j$-fold coloring of $G_{0}$ with small values of $j$ using $k$ colors are summarized in Table 3. They follow from Theorems 4, 5, 6, 7 .

For practical applications it is useful to consider $j$-fold coloring of graph $G_{[1,2]}$, especially with small $j$. Table 4 presents our results in coloring $G_{[1,2]}$ using method from Theorem 6. It appears that in this case the method from Theorem 7 does not give good results.

Acknowledgments We thank Professor Zbigniew Lonc, Zbigniew Walczak and Professor Jacek Wojciechowski for introducing us to the problem. 
Table 4 Applications of Theorem 6 for $G_{[1,2]}$ with small $j$

\begin{tabular}{llllll}
\hline$k=$ & 12 & 70 & 100 & 930 & 960 \\
\hline$j=$ & 1 & 6 & 9 & 84 & 87 \\
$k / j \approx$ & 12 & 11.67 & 11.11 & 11.07 & 11.03 \\
\hline
\end{tabular}

Open Access This article is distributed under the terms of the Creative Commons Attribution 4.0 International License (http://creativecommons.org/licenses/by/4.0/), which permits unrestricted use, distribution, and reproduction in any medium, provided you give appropriate credit to the original author(s) and the source, provide a link to the Creative Commons license, and indicate if changes were made.

\section{References}

1. Exoo, G.: $\varepsilon$-Unit distance graphs. Discrete Comput. Geom. 33, 117-123 (2005)

2. Falconer, K.J.: The realization of distances in measurable subsets covering $\mathbb{R}^{n}$. J. Comb. Theory, Ser. A 31, 187-189 (1981)

3. Fisher, D., Ullman, D.: The fractional chromatic number of the plane. Geombinatorics 2(1), 8-12 (1992)

4. Hadwiger, H.: Ungelöste Probleme. Elem. Math. 16, 103-104 (1961)

5. Hochberg, R., O’Donnell, P.: A large independent set in the unit distance graph. Geombinatorics 3(4), 83-84 (1993)

6. Ivanov, L.L.: On the chromatic numbers of $\mathbb{R}^{2}$ and $\mathbb{R}^{3}$ with intervals of forbidden distances. Electron. Notes Discrete Math. 29, 159-162 (2007)

7. Moser, L., Moser, W.: Solution for problem 10. Can. Bull. Math. 4, 187-189 (1961)

8. Nielsen, M.J.: Approximating monochromatic triangles in a two-colored plane. Acta Math. Hung. 74(4), 279-286 (1997)

9. Scheinerman, E.R., Ullman, D.H.: Fractional Graph Theory. Wiley, New York (2008)

10. Soifer, A.: The Mathematical Coloring Book. Springer, New York (2008)

11. Walczak, Z., Wojciechowski, J.M.: Transmission scheduling in packet radio networks using graph coloring algorithm, wireless and mobile communications. In: ICWMC '06 (2006)

12. Woodall, D.R.: Distances realized by sets covering the plane. J. Comb. Theory, Ser. A 74(4), 279-286 (1997) 